\title{
Transgenic expression of rice MYB102 (OsMYB102) delays leaf senescence and decreases abiotic stress tolerance in Arabidopsis thaliana
}

\author{
Weilan Piao ${ }^{1}$, Yasuhito Sakuraba ${ }^{1,2, *} \mathcal{E}$ Nam-Chon Paek ${ }^{1, *}$ \\ ${ }^{1}$ Department of Plant Science, Plant Genomics and Breeding Institute, and Research Institute of Agriculture and Life Sciences, Seoul \\ National University, Seoul 08826, Korea, ${ }^{2}$ Graduate School of Agricultural and Life Sciences, Biotechnology Research Center, The \\ University of Tokyo, Tokyo 113-8657, Japan
}

\begin{abstract}
MYB-type transcription factors (TFs) play important roles in plant growth and development, and in the rapid responses to unfavorable environmental conditions. We recently reported the isolation and characterization of a rice (Oryza sativa) MYB TF, OsMYB102, which is involved in the regulation of leaf senescence by downregulating abscisic acid (ABA) biosynthesis and the downstream signaling response. Based on the similarities of their sequences and expression patterns, OsMYB102 appears to be a homolog of the Arabidopsis thaliana AtMYB44 TF. Since AtMYB44 is a key regulator of leaf senescence and abiotic stress responses, it is important to examine whether AtMYB44 homologs in other plants also act similarly. Here, we generated transgenic Arabidopsis plants expressing OsMYB102 (OsMYB102-OX). The OsMYB102-OX plants showed a delayed senescence phenotype during dark incubation and were more susceptible to salt and drought stresses, considerably similar to Arabidopsis plants overexpressing AtMYB44. Real-time quantitative PCR (RT-qPCR) revealed that, in addition to known senescence-associated genes, genes encoding the ABA catabolic enzymes AtCYP707A3 and AtCYP707A4 were also significantly upregulated in OsMYB102$O X$, leading to a significant decrease in ABA accumulation. Furthermore, protoplast transient expression and chromatin immunoprecipitation assays revealed that OsMYB102 directly activated AtCYP707A3 expression. Based on our findings, it is probable that the regulatory functions of AtMYB44 homologs in plants are highly conserved and they have vital roles in leaf senescence and the abiotic stress responses. [BMB Reports 2019;
\end{abstract}

*Corresponding authors. Yasuhito Sakuraba, Tel: +82-2-880-4543; Fax: +82-2-877-4550; E-mail: sakuraba0425@gmail.com; NamChon Paek, Tel: +82-2-880-4543; Fax: +82-2-877-4550; E-mail: ncpaek@snu.ac.kr

https://doi.org/10.5483/BMBRep.2019.52.11.071

Received 15 March 2019, Revised 8 April 2019, Accepted 23 April 2019

Keywords: Abiotic stress, Arabidopsis thaliana, Leaf senescence, OsMYB102, Transcription factor
52(11): 653-658]

\section{INTRODUCTION}

Transcription factors (TFs) are involved in the regulation of gene expression and generally consist of two main domains, the DNA-binding and activation/repression domains (1). In plants, the MYB superfamily is one of the largest TF families, with approximately 198 members in Arabidopsis thaliana and 183 members in rice (Oryza sativa) (2). Plant MYB TFs regulate multiple biological processes, such as biotic/abiotic stress response, cell cycle, hormone signaling, and secondary metabolism (3-6). Some transcriptomic analyses have also indicated the importance of the MYB TFs during the onset of leaf senescence $(7,8)$. In Arabidopsis, AtMYBH was identified as a up-regulator of leaf senescence, with the atmybh mutants retaining leaf green color much longer and the AtMYBHoverexpressing lines exhibited an early leaf senescing phenotype during natural and dark-induced leaf senescence (9). By contrast, AtMYB44 is a down-regulator of leaf senescence, since Arabidopsis transgenics overexpressing AtMYB44 delayed leaf senescence and the atmyb44 mutants senesced precociously (10). In addition, AtMYB44 directly represses the transcription of senescence-associated genes (SAGs), including WRKY7O and ETHYLENE INSENSITIVE 2 (EIN2) $(11,12)$.

Although little is known about the senescence-associated MYB TFs in crop plants, we previously revealed that a rice MYB TF, OsMYB102, is involved the regulation of leaf senescence (13). A T-DNA-mediated activation-tagging line of OsMYB102 (osmyb102$D$ ) and OsMYB102-overexpressing transgenic plants showed a strong delayed senescence phenotype during both natural and artificially induced leaf senescence (13). In addition, OsMYB102 was found to directly activate the expression of OsCYP707A6, encoding an $A B A$ catabolic enzyme, and to indirectly repress the transcription of OsNAP and OsABF4, which encode TFs associated with the response to ABA signaling (13). OsMYB102 therefore appears to delay leaf yellowing by inhibiting both $A B A$ concentration and $\mathrm{ABA}$ signaling response. Through the genome-wide comparison of MYB TFs between Arabidopsis and

ISSN: 1976-670X (electronic edition)

Copyright (c) 2019 by the The Korean Society for Biochemistry and Molecular Biology

(c) This is an open-access article distributed under the terms of the Creative Commons Attribution Non-Commercial License (http://creativecommons.org/licenses/by-nc/4.0) which permits unrestricted non-commercial use, distribution, and reproduction in any medium, provided the original work is properly cited. 
rice, it revealed that OsMYB102 is a high candidate of AtMYB44 orthologue (14), and their R2R3 domains have $81 \%$ identity. As described above. AtMYB44 acts as a key regulator of leaf senescence and abiotic stress responses in Arabidopsis, but it is still largely unknown whether AtMYB44 homolog of other plant species act similarly.

In this study, we generated transgenic Arabidopsis plants expressing OsMYB102 (hereafter OsMYB102-OX) to examine whether OsMYB102 plays a similar role in the regulation of leaf senescence in both rice and Arabidopsis. We found that the OsMYB102-OX plants displayed a delayed leaf senescence phenotype during dark-induced senescence (DIS), and were hypersensitive to salinity and drought stresses. Using a transcriptomic analysis, we found that a number of genes related to $A B A$ metabolism and signaling were differentially expressed in OsMYB102-OX. Furthermore, we used chromatin immunoprecipitation (ChIP) and protoplast transactivation assays to reveal that OsMYB102 directly binds to the promoter of AtCYP707A3 to activate its transcription. We discuss the similarities and differences of the roles of OsMYB102 in rice and AtMYB44 in Arabidopsis.

\section{RESULTS}

Transgenic expression of OsMYB102 in Arabidopsis inhibits shoot growth

OsMYB102 is an R2R3-type MYB TF in rice and the closest homolog of AtMYB44 in Arabidopsis. The expression patterns of OsMYB102 and AtMYB44 are also similar: Their transcripts are abundant both in leaves and roots (10) (Supplementary Fig. S1), and are also strongly induced by ABA treatment $(13,14)$, during DIS (Supplementary Fig. S2) (13), and under high salinity and

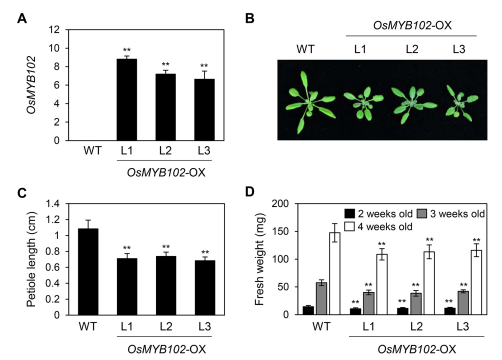

Fig. 1. The transgenic expression of OsMYB102 inhibits plant growth in Arabidopsis. Plants were grown under long-day (LD) conditions (16 $\mathrm{h}$ light $/ 8 \mathrm{~h}$ light). (A) The relative transcript levels of OsMYB102 in three-week-old Col-0 (WT) plants and three independent OsMYB102-OX lines were determined using RT-qPCR and normalized to the transcript levels of AtGAPDH. The mean and SD values were obtained from more than three biological samples. $(B, C)$ Visible phenotypes (B) and petiole lengths (C) of the WT and three independent OsMYB102-OX lines. (D) Fresh weights of two-, three-, and four-week-old WT and three independent OsMYB102-OX plants. Asterisks indicate a significant difference between WT and OsMYB102-OX plants (Student's t-test, $* P<0.05, * * P<0.01$ ). These experiments were repeated three times with similar results. drought stresses (Supplementary Fig. S3) (14). Thus, it is possible that OsMYB102 acts similar to AtMYB44 in Arabidopsis.

To examine the conserved function of OsMYB102 in Arabidopsis, we generated transgenic Arabidopsis plants that constitutively expressed OsMYB102 from a 35S:OsMYB102 construct. We used reverse transcription-quantitative real-time PCR (RT-qPCR) analyses to identify three independent OsMYB102-OX lines (Fig. 1A), and showed that, at the vegetative stage, these three OsMYB102-OX lines were all significantly smaller than the wild type (WT) and had shorter petioles (Fig. 1B, C). In addition, the fresh weights of the OsMYB102-OX plants were lower than the WT throughout their development (Fig. 1D), indicating that the constitutive expression of OsMYB102 has a negative effect on shoot growth in Arabidopsis.

\section{OsMYB102-OX plants have delayed leaf senescence}

OsMYB102 was previously shown to delay leaf senescence by inhibiting $A B A$ accumulation and signaling in rice (13); therefore, we examined the phenotype of the OsMYB102-OX plants during DIS. Four-week-old plants were transferred into complete darkness to induce artificial senescence. After $6 \mathrm{~d}$ of dark incubation (6 DDI), the WT leaves had turned yellow while the OsMYB102-OX leaves retained much of their greenness (Fig. 2A). Similar stay-green phenotypes were observed using rosette leaves detached from four-week-old OsMYB102-OX plants at 4 DDI (Fig. 2B). Consistent with the visible phenotype, the

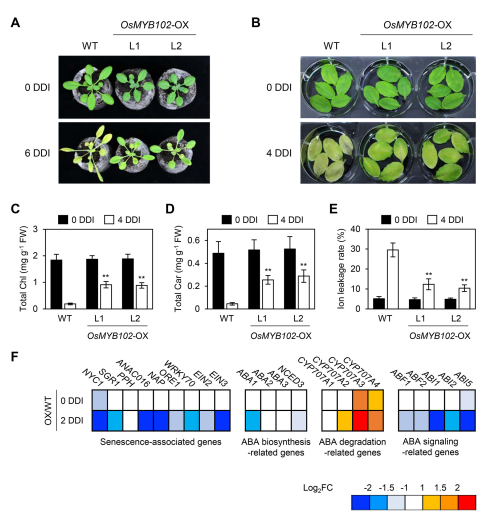

Fig. 2. The transgenic expression of OsMYB102 in Arabidopsis delays leaf senescence. (A) Four-week-old Col-0 (WT) and two independent OsMYB102-OX lines were transferred into complete darkness, and their visible phenotypes were observed at $6 \mathrm{~d}$ of dark incubation (DDI). (B-E) Changes in the leaf color (B), total chlorophyll (Chl) content (C), total carotenoid (Car) content (D), and membrane ion leakage rate $(\mathrm{E})$ in the detached rosette leaves of three-week-old WT and OsMYB102-OX plants at 0 and 4 DDI. The mean and SD values were obtained from more than five biological samples. Asterisks indicate a significant difference between the WT and OsMYB102-OX (Student's t-test, $* \mathrm{P}<0.05, * * \mathrm{P}<0.01$ ). (F) The relative expression levels (OsMYB102-OX/WT) of the senescence-associated genes (SAGs) and $\mathrm{ABA}$ metabolism- and signaling-related genes at 0 and $2 \mathrm{DDI}$ in OsMYB102-OX and the WT. These experiments were repeated twice with similar results. 
detached OsMYB102-OX leaves retained significantly more of their total chlorophyll (Chl) and total carotenoids (Car), while showing a lower ion leakage rate than the WT leaves (Fig. 2C-E). These findings indicate that OsMYB102 can delay leaf senescence in Arabidopsis, similar to its function in rice.

To understand how OsMYB102 delays leaf senescence in Arabidopsis, we first examined the expression patterns of the SAGs in the OsMYB102-OX plants during DIS (Fig. 2F). In addition, we checked the expression patterns of genes related to $A B A$ biosynthesis, degradation, and signaling , since OsMYB102 mainly affected the expression of the ABA-associated genes in rice (13). At 2 DDI, several SAGs, such as NON-YELLOW COLORING 1 (AtNYC1), encoding a Chl catabolic enzyme (15); AtNAP and AtANAC016, encoding senescence-associated NAC TFs (16, 17); and EIN3, encoding a key TF in ethylene-induced leaf senescence (18), were all significantly downregulated in the OsMYB102-OX leaves. The $\mathrm{ABA}$ signaling-related genes, including $A B A$ INSENSITIVE 1 (AtABI1) and AtABI5, were also downregulated, while the $A B A$ degradation-related genes (AtCYP707A3 and AtCYP707A4) were significantly upregulated in the OsMYB102-OX leaves. Remarkably, the expression levels of AtCYP707A3 and AtCYP707A4 in OsMYB102-OX were higher than they were in the $\mathrm{WT}$, even at $0 \mathrm{DDI}$. Taken together, these results suggest that the OsMYB102 TF functions to delay leaf senescence in Arabidopsis by

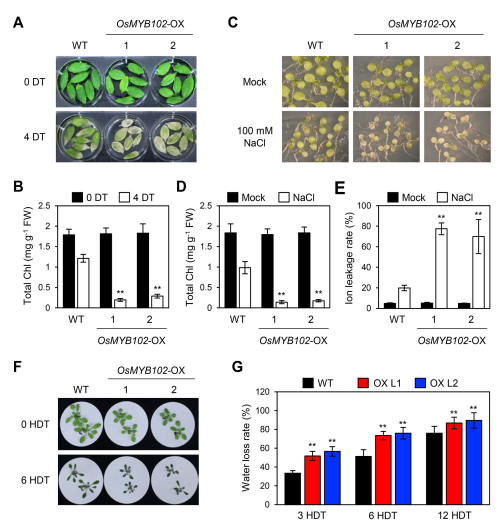

Fig. 3. The transgenic expression of OsMYB102 increases susceptibility to high salinity and drought stresses in Arabidopsis. (A) Rosette leaves detached from four-week-old Col-0 (WT) and two independent OsMYB102-OX lines were transferred into $3 \mathrm{mM}$ MES buffer ( $\mathrm{pH}$ 5.8) containing $150 \mathrm{mM} \mathrm{NaCl}$, and the changes of visible phenotypes (A) and total chlorophyll (Chl) contents (B) were examined before and after $4 \mathrm{~d}$ of treatment (DT). (C-E) The 5-d-old WT and two independent OsMYB102-OX lines grown on MS agar were transferred into MS agar containing $100 \mathrm{mM} \mathrm{NaCl}$. The visible phenotypes (C), total Chl contents (D), and membrane ion leakage rates (E) were examined at 3 DT. (F, G) Four-week-old WT and two independent OsMYB102-OX lines (L1, L2) were placed on dry filter paper and dehydrated for indicated hours. The phenotypes were observed at 6 $\mathrm{h}$ of dehydration treatment (6 HDT) and the water loss was determined at 3, 6, and 12 HDT. Mean and SD values were obtained from more than five biological replicates (Student's $t$-test, $* P<0.05$, $* * P<0.01)$. modulating the expression of a number of SAGs and $\mathrm{ABA}$ degradation- and signaling-related genes.

\section{OsMYB102-OX plants have decreased abiotic stress tolerance}

$A B A$ is a phytohormone with a vital role in the regulation of abiotic stress responses (19). Our finding that a number of genes involved in ABA metabolism and signaling were differentially expressed in the OsMYB102-OX leaves during DIS led us to examine the phenotype of the OsMYB102-OX plants under abiotic stress conditions, such as high salinity and drought stresses. When placed in Murashige and Skoog (MS) medium containing $150 \mathrm{mM} \mathrm{NaCl}$, the detached rosette leaves from the OsMYB102-OX plants became necrotic much faster than the WT leaves (Fig. 3A), resulting in a lower Chl content (Fig. 3B). A similar phenotype was also observed in young seedlings subjected to salt stress; when 5-d-old seedlings grown on MS agar plates were transferred to plates containing $100 \mathrm{mM} \mathrm{NaCl}$, the OsMYB102-OX seedlings were hypersensitive to the high salinity stress (Fig. 3C). Consistent with the visible phenotype, the OsMYB102-OX seedlings had a lower Chl content (Fig. 3D) and a higher ion leakage rate (Fig. 3E).

To examine the effect of OsMYB102 on drought tolerance in

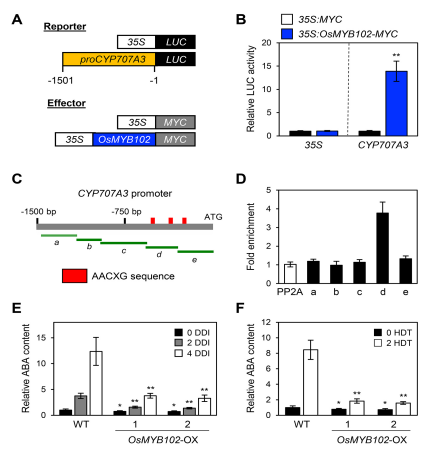

Fig. 4. The transgenic expression of OsMYB102 inhibits $A B A$ accumulation by upregulating AtCYP707A3 transcription. (A) Reporter and effector constructs used in the transactivation assay. Each construct also contained the NOS terminator (not shown). (B) The activation of AtCYP707A3 promoters $(-1501$ to -1 from the start codon) by the OsMYB102-Myc protein in a protoplast transient expression assay. The 35S promoter was used as the negative control. (C) The positions of the AACXG binding motif (red vertical lines) in the promoter of AtCYP707A3, and the promoter fragments used for the ChIP assay (green horizontal lines). (D) The in planta binding affinity of OsMYB102 to the promoter region of AtCYP707A3, examined using ChIP assays. The OsMYB102-Myc fusion protein was transiently expressed in protoplasts isolated from three-week-old Col-0 (WT) rosette leaves. The fold-enrichment of the promoter fragments was measured using an immunoprecipitation with an anti-Myc antibody. PP2A was used as the negative control. (E, F) Relative ABA contents in the leaves of the WT and two independent OsMYB102-OX lines during DIS (E) or dehydration (F). Mean and SD values were obtained from more than four biological samples. Asterisks indicate a significant difference from the WT or the negative control (Student's t-test, $* P<0.05, * * P<0.01)$. 
Arabidopsis, the whole plants of WT and two independent OsMYB102-OX lines were transferred onto dry filter paper. After $6 \mathrm{~h}$ of the drying treatment $(6 \mathrm{HDT})$, the OsMYB102-OX plants were almost completely wilted, while the WT plants seemed to have retained some freshness (Fig. 3F). Consistent with the visible phenotypes, the OsMYB102-OX plants lost significantly more of their relative fresh weights than the WT (Fig. 3G). These results demonstrate that the transgenic expression of OsMYB102 enhances plant susceptibility to abiotic stresses, including salt and drought stresses, which is probably caused by OsMYB102mediated modulation of a wide range of genes related to $A B A$ accumulation and signaling.

\section{OsMYB102 directly binds to the promoter of AtCYP707A3} and induces its transcription in Arabidopsis

In rice, OsMYB102 inhibits $A B A$ accumulation by directly inducing the expression of OsCYP707A6, which encodes an ABA catabolic enzyme (13). In this study, we found that AtCYP707A3 and AtCYP707A4, the Arabidopsis homologs of OsCYP707A6, were also upregulated in the OsMYB102-OX plants (Fig. 2F). We previously showed that OsMYB102 has the capacity to bind to the AACXG consensus sequence, which is also known to be the binding motif of the closest OsMYB102 homolog in Arabidopsis, AtMYB44 $(13,20)$. We therefore examined the promoters of AtCYP707A3 and AtCYP707A4 $(-1500$ to -1 from the start codon). We found that the promoter of AtCYP707A3 has four AACXG sequences (Fig. 4A), but the AtCYP707A4 promoter does not contain this motif.

We used a protoplast transient expression assay to determine the activity of OsMYB102 on the induction of AtCYP707A3 transcription. For the reporter construct, the promoter of AtCYP707A3 ( -1502 to -1 from the start codon) was fused with the luciferase (LUC) reporter, while 35S:OsMYB102-MYC was used as the effector construct (Fig. 4B). We found that the LUC activity of protoplasts expressing proAtCYP707A3:LUC increased when they were co-transfected with the effector plasmid 35S:OsMYB102-MYC (Fig. 4C). Next, with the Arabidopsis protoplasts expressing OsMYB102-MYC, we investigated the binding activity of OsMYB102 into the promoter region of AtCYP707A3. By a ChIP assay, we found that OsMYB102 bound to AtCYP707A3 promoter region d, which contains an AACXG motif (Fig. 4D), indicating that OsMYB102 directly activates AtCYP707A3 transcription in Arabidopsis.

To examine the effect of the activation of AtCYP707A3 transcription by OsMYB102, we measured the ABA contents in the WT and two OsMYB102-OX lines during DIS and dehydration. The ABA levels in the OsMYB102-OX lines were lower than those in the WT even under normal growth conditions, although this difference was magnified during the DIS and dehydration treatments (Fig. 4E, F), indicating that overexpression of OsMYB102 down-regulates ABA accumulation in Arabidopsis, at least in part by directly inducing the expression of AtCYP707A3.

\section{DISCUSSION}

In this study, we showed that transgenic Arabidopsis plants expressing OsMYB102 have a strong delayed-senescence phenotype during DIS and were more susceptible to salinity and drought stresses (Figs. 2, 3). These phenotypes of OsMYB102-OX plants resembled those of Arabidopsis transgenics overexpressing AtMYB44 which is the sequence homolog and probable functional homolog of OsMYB102 $(10,21)$. In addition, a delayed senescence phenotype was also observed in the rice osmyb102-D mutant and rice OsMYB102-OX plants (13). The transgenic expression of OsMYB102 inhibited the accumulation of ABA in Arabidopsis (Fig. 4), and ChIP and protoplast transient expression assays suggest that OsMYB102 directly activates the transcription of AtCYP707A3 (Fig. 4) which is the homolog of OsCYP707A6 (13). ABA promotes leaf senescence and also enhances tolerance to abiotic stresses $(19,22)$; therefore, probably the decrease of $A B A$ accumulation by overexpression of OsMYB102 led to delayed senescence phenotype and a decrease in plant tolerance to abiotic stresses such as salinity and drought (Figs. 2, 3).

In Arabidopsis, OsMYB102-OX plants also showed shorter petiole and semi-dwarf phenotypes under normal growth conditions (Fig. 1), similar to AtMYB44-OX (21). It was previously reported that Arabidopsis transgenics overexpressing AtCYP707A3 and an ABA-deficient mutant aba2-2 showed a semi-dwarf phenotype (23), similar to OsMYB102-OX (Fig. 1). Such a semidwarf phenotype is one of the typical phenotypes of ABAdeficient mutants, and probably it is caused by pleiotropic effects of $\mathrm{ABA}$ deficiencies, such as decrease of stomata closure rate when it is necessary. Collectively, these results strongly indicate that the physiological roles of OsMYB102 and its homologs are highly conserved, and that these genes may act as key regulators of $\mathrm{ABA}$ metabolism and signaling in various plant species.

We also identified some differences between Arabidopsis and rice plants expressing OsMYB102. We found that the Arabidopsis OsMYB102-OX plants were hypersensitive to high salinity and drought stresses (Fig. 3); however, this was not previously observed in rice osmyb102-D or OsMYB102-OX plants (13). So far, it is not clear why the overexpression of OsMYB102 in rice does not alter its tolerance to abiotic stresses. One possibility is that, in rice, OsMYB102 has an important role in regulating other signaling pathways, diminishing the effect of this TF on abiotic stress tolerance. Further phenome and transcriptome analyses are necessary for elucidating the transcriptional regulatory networks mediated by OsMYB102.

\section{MATERIALS AND METHODS}

\section{Plant materials and growth conditions}

Arabidopsis thaliana Columbia-0 (Col-0) seeds were obtained from the Arabidopsis Biological Resource Center (ABRC). The Arabidopsis plants were grown on soil at $22-24^{\circ} \mathrm{C}$ in long-day conditions ( $16 \mathrm{~h}$ light/8 $\mathrm{h}$ dark) in a growth room equipped 
with cool-white fluorescent lights $\left(100 \mu \mathrm{mol} \mathrm{m} \mathrm{m}^{-2} \mathrm{~s}^{-1}\right)$. DIS was induced as previously described (24). Briefly, detached leaves were kept in 3 mM MES (pH 5.8) buffer in full darkness, or whole plants were transferred into full darkness.

\section{Plasmid construction and transformation}

The OsMYB102 cDNA was amplified using RT-PCR with gene-specific primers (Supplementary Table S1) and sub-cloned into the pCR8/GW/TOPO plasmid, after which it was ligated into the pEarleyGate 203 gateway binary vector containing the $35 \mathrm{~S}$ promoter (25). The recombinant plasmids were transformed into Agrobacterium tumefaciens strain GV3101. The Agrobacteriummediated transformation into WT (Col-0) was performed using the floral-dip method (26). Transgenic $\mathrm{T}_{0}$ seedlings were selected on the $0.5 \times$ MS phytoagar medium supplemented with $2 \mathrm{mM}$ MES buffer ( $\mathrm{pH}$ 5.8) and $12 \mathrm{mg} \mathrm{l}^{-1}$ phosphinothricin. The phosphinothricin-resistant seedlings were planted and reselected to obtain the final $T_{2}$ homozygous lines.

\section{Reverse transcription-quantitative real-time PCR (RT-qPCR)} For the RT-qPCR analysis, the total RNA was isolated from Arabidopsis rosette leaves using the TRIzol reagent (Thermo Fisher Scientific). The first-strand cDNAs were prepared using 5 $\mu \mathrm{g}$ total RNA in a $25-\mu$ l reaction volume using M-MLV reverse transcriptase and oligo(dT) $)_{15}$ primers (Promega), then diluted with water to $100 \mu \mathrm{l}$. The $20-\mu \mathrm{l}$ qPCR mixture contained $1 \mu \mathrm{l}$ CDNA, $10 \mu \mathrm{l} 2 \times$ SYBR Green PCR Master Mix (Qiagen), and $0.25 \mathrm{mM}$ each of the forward and reverse gene-specific primers (Supplementary Table S1). The qPCR analysis was carried out using a LightCycler 480 (Roche Diagnostics). To determine the relative expression, the mRNA abundance of each gene was normalized to that of AtGAPDH (At1g16300).

\section{Chlorophyll (Chl) and carotenoid (Car) quantification}

To measure total $\mathrm{Chl}$ and Car concentrations, the pigments were extracted from leaf tissues using $80 \%$ ice-cold acetone. The concentrations were determined using spectrophotometry, as described previously (27).

\section{Measurement of ion leakage rates}

Ion leakage was measured as described previously (28). Briefly, the membrane leakage was determined by measuring the loss of electrolytes (or ions) from the rosette leaves or seedlings. Ten leaves from each treatment were immersed in $6 \mathrm{ml}$ of $0.4 \mathrm{M}$ mannitol at room temperature and gently shaken for $3 \mathrm{~h}$. The initial conductivity of the solution was then measured with a conductivity meter (CON6 Meter; LaMotte). After a 20-min incubation at $85^{\circ} \mathrm{C}$, the total conductivity of the sample was determined. The rate of ion leakage was expressed as the percentage of initial conductivity divided by the total conductivity.

\section{Stress treatments}

The detached leaves and seedlings were exposed to a salt treatment as previously described (29). The dehydration treatment on dry filter paper was carried out following a previously described protocol (27).

\section{Chromatin Immunoprecipitation assay}

Plants expressing OsMYC102-MYC were grown in continuous white light for $7 \mathrm{~d}$ before being cross-linked for $20 \mathrm{~min}$ with $1 \%$ formaldehyde under vacuum. The chromatin complexes were isolated and sonicated as previously described (30), using an anti-MYC polyclonal antibody (Abcam) and Protein A agarose/ salmon sperm DNA (Millipore) for the immunoprecipitation. After reverse cross-linking and protein digestion, the DNA was purified using a QIAquick PCR Purification Kit (Qiagen).

\section{Co-transfection and transient expression in Arabidopsis protoplasts}

To construct the reporter plasmids containing the $L U C$ reporter gene under the control of $A t C Y P 707 A 3$ promoter, the promoter fragment of AtCYP707A3 ( -1501 to -1 from the start codon) was cloned into the pGreenll-0579 vector, which combined the $L U C$ reporter sequence with the gene of interest at its C-terminus. The plasmid containing 35S:OsMYC102-MYC was previously generated (13). The reporter $(4 \mu \mathrm{g})$ and effector plasmids $(8 \mu \mathrm{g})$ were co-transfected into $5 \times 10^{4}$ Arabidopsis protoplasts by polyethylene glycol-mediated transfection (31). The transfected protoplasts were then suspended in protoplast culture medium (0.4 mM mannitol, $15 \mathrm{mM} \mathrm{MgCl}_{2}$, and $4 \mathrm{mMMES}, \mathrm{pH}$ 5.8) and kept in the dark for $16 \mathrm{~h}$. The LUC activity in each cell lysate was determined using the Luciferase Assay System Kit (Promega).

\section{Quantification of ABA contents}

To determine the $\mathrm{ABA}$ contents, three-week-old plants were dark-incubated or dehydrated for the indicated times, then collected and weighed. The leaves were ground in liquid nitrogen and homogenized with $80 \%$ methanol containing 1 $\mathrm{mM}$ butylated hydroxytoluene, then incubated overnight at $4^{\circ} \mathrm{C}$ to extract the endogenous $\mathrm{ABA}$. The $\mathrm{ABA}$ contents were analyzed using the ABA ELISA Kit (MyBiosource).

\section{ACKNOWLEDGEMENTS}

This work was supported by the Cooperative Research Program for Agricultural Science \& Technology Development (PJ013130), Rural Development Administration, Republic of Korea, and the Basic Science Research Program through the National Research Foundation (NRF) of Korea, funded by the Ministry of Education (NRF-2017R1A2B3003310).

\section{CONFLICTS OF INTEREST}

The authors have no conflicting interests. 


\section{REFERENCES}

1. Ptashne $M$ and Gann AA (1990) Activators and targets. Nature 346, 329-331

2. Yanhui C, Xiaoyuan $\mathrm{Y}$, Kun $\mathrm{H}$ et al (2006) The MYB transcription factor superfamily of Arabidopsis: expression analysis and phylogenetic comparison with the rice MYB family. Plant Mol Biol 60, 107-124

3. Cominelli E and Tonelli C (2009) A new role for plant R2R3-MYB transcription factors in cell cycle regulation. Cell Res 19, 1231-1232

4. Liu J, Osbourn A and Ma P (2015) MYB Transcription Factors as Regulators of Phenylpropanoid Metabolism in Plants. Mol Plant 8, 689-708

5. Baldoni E, Genga A and Cominelli E (2015) Plant MYB transcription factors: their role in drought response mechanisms. Int J Mol Sci 16, 15811-15851

6. Byun MY, Cui LH, Lee H and Kim WT (2018) Telomere association of Oryza sativa telomere repeat-binding factor like 1 and its roles in telomere maintenance and development in rice, Oryza sativa L. BMB Rep 51, 578-583

7. Balazadeh S, Riano-Pachon DM and Mueller-Roeber B (2008) Transcription factors regulating leaf senescence in Arabidopsis thaliana. Plant Biol (Stuttg) 10 Suppl 1, 63-75

8. Moschen S, Bengoa Luoni S, Di Rienzo JA et al (2016) Integrating transcriptomic and metabolomic analysis to understand natural leaf senescence in sunflower. Plant Biotech J 14, 719-734

9. Huang CK, Lo PC, Huang LF, Wu SJ, Yeh CH and Lu CA (2015) A single-repeat MYB transcription repressor, MYBH, participates in regulation of leaf senescence in Arabidopsis. Plant Mol Biol 88, 269-286

10. Jaradat MR, Feurtado JA, Huang D, Lu Y and Cutler AJ (2013) Multiple roles of the transcription factor AtMYBR1/AtMYB44 in ABA signaling, stress responses, and leaf senescence. BMC Plant Biol 13, 192

11. Liu R, Chen L, Jia $Z$ et al (2011) Transcription factor AtMYB44 regulates induced expression of the ETHYLENE INSENSITIVE 2 gene in Arabidopsis responding to a harpin protein. Mol Plant Microbe Interac 24, 377-389

12. Besseau S, Li J and Palva ET (2012) WRKY54 and WRKY70 co-operate as negative regulators of leaf senescence in Arabidopsis thaliana. J Exp Bot 63, 2667-2679

13. Piao W, Kim SH, Lee BD, An G, Sakuraba Y and Paek NC (2018) The rice transcription factor OsMYB102 delays leaf senescence by downregulating abscisic acid accumulation and signaling. J Exp Bot. DOI: 10.1093/jxb/erz095

14. Katiyar A, Smita S, Lenka SK, Rajwanshi R, Chinnusamy V, and Bansal KC (2012) Genome-wide classification and expression analysis of MYB transcription factor families in rice and Arabidopsis. BMC Genomics 13, 544

15. Horie $Y$, Ito H, Kusaba M, Tanaka R and Tanaka A (2009) Participation of chlorophyll b reductase in the initial step of the degradation of light-harvesting chlorophyll a/b-protein complexes in Arabidopsis. J Biol Chem 284, 17449-17456

16. Guo $Y$ and Gan S (2006) AtNAP, a NAC family transcription factor, has an important role in leaf senescence. Plant J 46, 601-612
17. Kim YS, Sakuraba Y, Han SH, Yoo SC and Paek NC (2013) Mutation of the Arabidopsis NAC016 transcription factor delays leaf senescence. Plant Cell Physiol 54, 1660-1672

18. Li Z, Peng J, Wen X and Guo H (2013) ETHYLENE-INSENSITIVE 3 is a senescence-associated gene that accelerates agedependent leaf senescence by directly repressing miR164 transcription in Arabidopsis. Plant Cell 25, 3311-3328

19. Qin F, Shinozaki K and Yamaguchi-Shinozaki K (2011) Achievements and challenges in understanding plant abiotic stress responses and tolerance. Plant Cell Physiol 52, 1569-1582

20. Jung C, Kim YK, Oh NI et al (2012) Quadruple 9-mer-based protein binding microarray analysis confirms AACnG as the consensus nucleotide sequence sufficient for the specific binding of AtMYB44. Mol Cells 34, 531-537

21. Jung $C$, Seo JS, Han SW et al (2008) Overexpression of AtMYB44 enhances stomatal closure to confer abiotic stress tolerance in transgenic Arabidopsis. Plant Physiol 146, 623-635

22. Cho SK, Ryu MY, Kim JH et al (2017) RING E3 ligases: key regulatory elements are involved in abiotic stress responses in plants. BMB Rep 50, 393-400

23. Umezawa T, Okamoto M, Kushiro T et al (2006) CYP707A3, a major $\mathrm{ABA}$ 8'-hydroxylase involved in dehydration and rehydration response in Arabidopsis thaliana. Plant J 46, 171-182

24. Sakuraba Y, Park SY, Kim YS et al (2014) Arabidopsis STAY-GREEN2 is a negative regulator of chlorophyll degradation during leaf senescence. Mol Plant 7, 1288-1302

25. Earley KW, Haag JR., Pontes O et al (2006) Gatewaycompatible vectors for plant functional genomics and proteomics. Plant J 45, 616-629

26. Zhang X, Henriques R, Lin SS, Niu QW and Chua NH (2006) Agrobacterium-mediated transformation of Arabidopsis thaliana using the floral dip method. Nat Protoc 1, 641-646

27. Porra RJ, Thompson WA and Kriedemann PE (1989) Determination of accurate extinction coefficients and simultaneous equations for assaying chlorophylls $a$ and $b$ extracted with four different solvents: verification of the concentration of chlorophyll standards by atomic absorption spectroscopy. Bioch Biophy Acta 975, 384-394

28. Sakuraba Y, Kim YS, Han SH, Lee BD and Paek NC (2015) The Arabidopsis transcription factor NAC016 promotes drought stress responses by repressing AREB1 transcription through a trifurcate feed-forward regulatory loop involving NAP. Plant Cell 27,1771-1787

29. Sakuraba Y, Bulbul S, Piao WL, Choi G and Paek NC (2017) Arabidopsis EARLY FLOWERING 3 increases salt tolerance by suppressing salt stress response pathways. Plant J 92, 1106-1120

30. Sakuraba Y, Kanno S, Mabuchi A, Monda K, Iba K and Yanagisawa S (2018) A phytochrome B-mediated regulatory mechanism of phosphorus acquisition. Nat Plants 4 1089-1101

31. Yoo SD, Cho YH and Sheen J (2007) Arabidopsis mesophyll protoplasts: a versatile cell system for transient gene expression analysis. Nat Protoc 2, 1565-1572 